\title{
First heavy ions at LHC - inclusive production, correlations and heavy flavours
}

\section{Luciano Ramello*}

Dipartimento di Scienze e Tecnologie Avanzate dell'Università del Piemonte Orientale and INFN, V.le T. Michel 11, 15121 Alessandria, Italy

E-mail: ramellodto.infn.it

This review covers the heavy ion programmes of three experiments at LHC: ALICE, ATLAS and CMS. The capabilities of the experiments for inclusive particle production, correlations and heavy flavours are presented, and the prospective results from the upcoming first heavy ion run of LHC are discussed.

\footnotetext{
* Speaker.
} 


\section{Introduction}

The Large Hadron Collider at CERN, after a long construction [1] and hardware commissioning [2, 3] phase, is finally starting operations ${ }^{1}$. The first heavy ion $(\mathrm{Pb}-\mathrm{Pb})$ run is currently scheduled at the end of 2010, after the proton-proton run; the initial center-of-mass energy could be $\approx 4 \mathrm{~A} \mathrm{TeV}$ (the nominal energy is $5.5 \mathrm{~A} \mathrm{TeV}$ ), a factor 20 above maximum RHIC energy. During 9 years of operations, experiments at RHIC have produced a wealth of results [ 4 ] on QCD matter at high temperature and density; open questions on the properties of the Quark Gluon Plasma (QGP) are expected to be answered by the continuation of the RHIC programme and, above all, by the LHC heavy ion programme.

Three large LHC experiments have prepared a physics programme with heavy ions, namely ALICE [5, 6, 7], ATLAS [8, 9] and CMS [10, 11]. In this review, the heavy ion programmes of the three experiments are presented (for recent presentations by each experiment, see [12, $13,14,15]$ ), covering the topics of inclusive particle production, correlations and heavy flavours; prospective results from the upcoming first heavy ion run of LHC are discussed. The topics of high $p_{T}$ hadrons, photons, jets and quarkonia are discussed in another review [16]. The previous review was given in [17].

The three LHC experiments have been designed with different constraints, therefore a brief discussion of their capabilities for heavy ion collisions is in order. ALICE is the only experiment designed for heavy ion physics and is therefore covering all the relevant observables [巨, 5]; it has very robust tracking with many points per track, the lowest material budget in its barrel region $(|\eta|<0.9)$, powerful particle identification (PID) over a large $p_{T}$ range and excellent vertexing for charm and beauty identification. ATLAS, although primarily designed for pp collisions, has excellent capabilities for $\mathrm{Pb}-\mathrm{Pb}$ collisions [8] concerning (for the topics reviewed here) charged hadron and muon production in the barrel $(|\eta|<2.5)$ with 3 layers of Si pixels, the TRT and the SCT detectors, and also total transverse energy measurement. CMS is also primarily designed for pp collisions, but has excellent capabilities for $\mathrm{Pb}-\mathrm{Pb}$ collisions [10] as well, including tracking in the barrel $(|\eta|<2.5)$ with Si pixels and strips (charged hadrons and muons), calorimetry up to $\eta=5$ and specialized forward detectors. The next three sections will deal with inclusive particle production, correlations and open heavy flavours.

\section{Inclusive production and identified particle $p_{T}$ spectra}

The first observables at $\mathrm{LHC}$, accessible with a few thousand to tens of thousand $\mathrm{Pb}-\mathrm{Pb}$ collisions, will be those regarding inclusive particle production, namely: charged particle multiplicity and density, transverse momentum spectra of charged particles; as more statistics is collected, identified particle $p_{T}$ spectra of stable hadrons and resonances, as well as ratios between yields of different species, will become accessible as well. The centrality of collisions will be determined on an event-by-event basis by all experiments: ALICE [6] will use zero-degree proton and neutron calorimeters as well as barrel multiplicity, ATLAS [18] will use total transverse energy, zero-degree energy and the signals from the LUCID detector, while CMS [10] will use transverse

\footnotetext{
${ }^{1}$ In fact, just three days after the conclusion of this Symposium, first proton-proton collisions at the injection centerof-mass energy of $900 \mathrm{GeV}$ were obtained in the four large experiments, on November 23, 2009.
} 

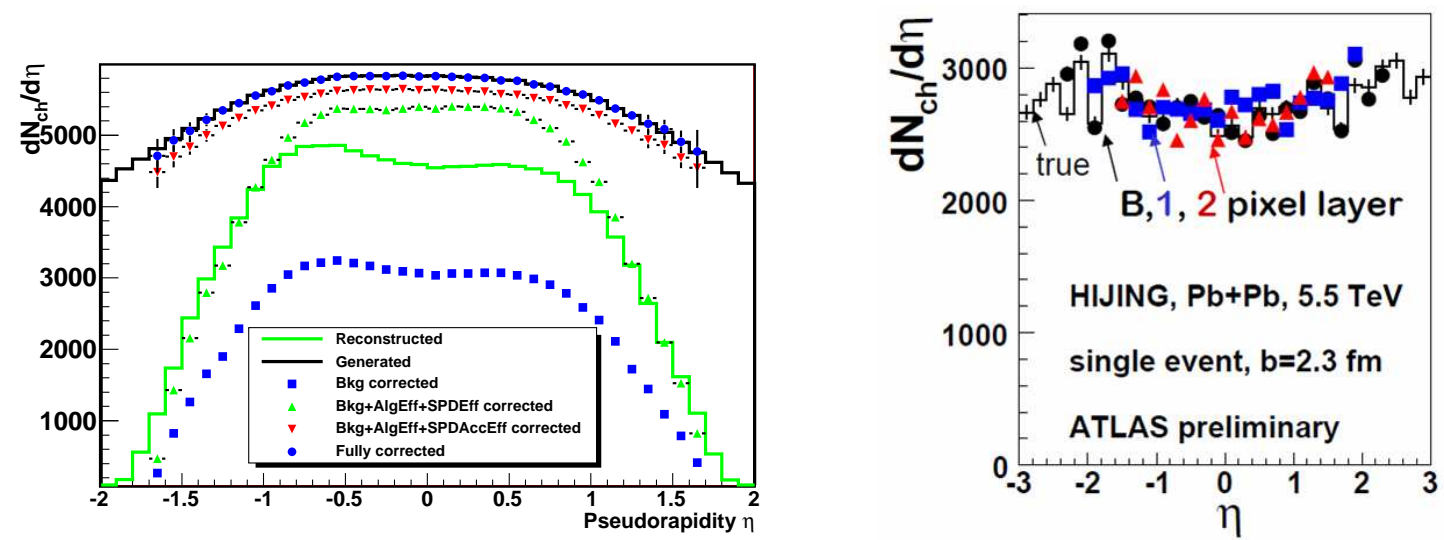

Figure 1: Left: pseudorapidity density for $\mathrm{Pb}-\mathrm{Pb}$ central events simulated and reconstructed in ALICE using tracklets; right: generated (line) and reconstructed (symbols) $d N_{c h} / d \eta$ of a single central $\mathrm{Pb}-\mathrm{Pb}$ event in the three layers of the ATLAS pixel detector.

energy in forward $(3<\eta<6.7)$ calorimeters as well as neutron zero-degree energy. The centrality could then be expressed e.g. as the number of participant nucleons, $N_{\text {part }}$.

The charged particle pseudorapidity density normalized to the number of participant pairs, $\left(d N_{c h} / d \eta\right) /\left(N_{\text {part }} / 2\right)$, as a function of nucleon-nucleon center-of-mass energy is a key observable to assess the validity of the gluon saturation model (see e.g. [19]) proposed for heavy ion collisions. All three experiments are well equipped for the measurement of $d N_{c h} / d \eta$ : ALICE will use tracklets in the two layers of pixel detectors $(-1.7<\eta<1.7)$ as shown in Fig. 1 (left), to be complemented by the Forward Multiplicity Detector, reaching eventually $\approx 8$ units in $\eta$; ATLAS will use the first three pixel layers, as shown in Fig. 1] (right) for a single event, while CMS [10] will use both hits in the first layer of pixels and tracklets. All experiments will apply corrections for secondary particles, fake combinations, detector acceptance and efficiency and trigger efficiency (see e.g. subsequent steps from reconstructed to fully corrected $d N_{c h} / d \eta$ in Fig. 11 left).

Transverse momentum spectra of charged particles provide information on the hadronic system at kinetic freezeout; in order to provide an accurate measurement of the yields of various particle species, which can be related to system parameters as temperature and baryonic chemical potential at the chemical freezeout, efficient tracking down to low $p_{T}$ is crucial. CMS has developed a hit triplet algorithm [10, 20, 21] which uses three layers of pixels and provides in central $\mathrm{Pb}-\mathrm{Pb}$ collisions pion reconstruction for $p_{T}$ as low as $0.2 \mathrm{GeV} / \mathrm{c}$ and a flat efficiency of $\approx 90 \%$ above $0.4 \mathrm{GeV} / \mathrm{c}$, together with a reasonably low fake rate. ATLAS has obtained [14] for simulated central $\mathrm{Pb}-\mathrm{Pb}$ collisions a negligible fake rate and an efficiency of $\approx 70 \%$ above $1 \mathrm{GeV} / \mathrm{c}$. ALICE will use as a baseline tracking in ITS and TPC [7] which shows a good performance in central $\mathrm{Pb}-\mathrm{Pb}$ collisions above $0.2 \mathrm{GeV} / \mathrm{c}$, the efficiency being limited at $\approx 90 \%$ at high $p_{T}$ due to TPC dead zones (a complementary approach which uses ITS standalone tracking is available).

Spectra of identified $\pi, K$ and $p$ at low $p_{T}$ will be obtained in ALICE using the momentum and $\mathrm{dE} / \mathrm{dx}$ information from the TPC [7], by building for each $p_{T}, \eta$ bin the histogram of (signal $-\mathrm{BB}_{\pi}$ )/BB $\mathrm{BB}_{\pi}$ (where $\mathrm{BB}_{\pi}$ is the Bethe-Bloch $\mathrm{dE} / \mathrm{dx}$ parametrization of the TPC signal for pions) and extracting the yields from a multi-gaussian fit. At higher momentum up to 5-6 GeV/c, the 

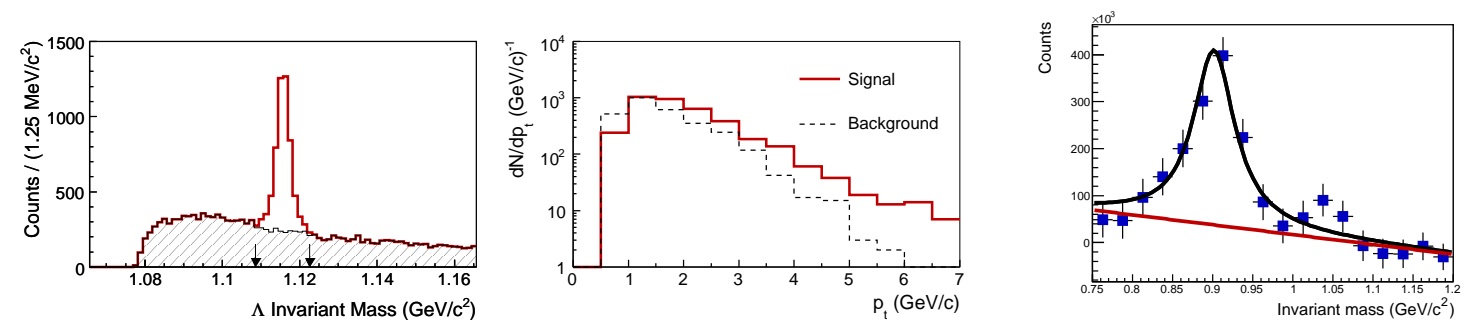

Figure 2: Left: $\Lambda$ invariant mass spectrum as simulated and reconstructed in ALICE; center: $p_{T}$ spectrum of $\Lambda$ 's reconstructed in ALICE; right: $K^{* 0}$ invariant mass spectrum as simulated and reconstructed in ALICE.

Time-Of-Flight (TOF) signal together with TPC tracking will be used in ALICE [6], and even at lower $p_{T}$ the TOF will help the TPC by resolving ambiguities at places where the TPC $\mathrm{dE} / \mathrm{dx}$ bands cross each other. The Transition Radiation Detector (TRD) will be used for electron identification in ALICE. In CMS, separation of $\pi, K$ and $p$ up to $1 \mathrm{GeV} / \mathrm{c}$ and of $(\pi+K)$ from $p$ up to $2 \mathrm{GeV} / \mathrm{c}$ will be possible using $\mathrm{dE} / \mathrm{dx}$ in the three pixel layers [10].

Enhancement of the strangeness production is a historical signature of QGP formation [22]. ALICE has developed topological identification of strange hadrons [6, 23] based on ITS (vertexing and impact parameter) and TPC (tracking): in a standard $\mathrm{Pb}-\mathrm{Pb}$ run of 1 month (10 million central $\mathrm{Pb}-\mathrm{Pb}$ collisions) the yields and $p_{T}$ spectra of $K_{s}^{0}$ (also charged $K$ ) and $\Lambda(\bar{\Lambda})$ can be measured accurately (see e.g. Fig. 目 left and center) up to $p_{T}$ of $13-15 \mathrm{GeV} / \mathrm{c}$, while for $\Xi(\bar{\Xi})$ and $\Omega(\bar{\Omega})$ the statistical limit is $\approx 9-12 \mathrm{GeV} / \mathrm{c}$. CMS will be able to extract the inclusive yield of $K_{s}^{0}$ 's and $\Lambda$ 's in $\mathrm{Pb}-\mathrm{Pb}$ collisions.

Resonances, both strange and non-strange, provide crucial information on the medium due to their range of lifetimes (from $1.3 \mathrm{fm} / \mathrm{c}$ for the $\rho$ to $45 \mathrm{fm} / \mathrm{c}$ for the $\phi$ ) in comparison with expected QGP lifetimes at LHC (at least $10 \mathrm{fm} / \mathrm{c}$ ). ALICE simulations [6], based on different PID and combinatorial background subtraction approaches, provide an estimated invariant mass resolution between 1.5 and $3 \mathrm{MeV} / \mathrm{c}^{2}$ for most resonances and statistical limits on the $p_{T}$ spectra ranging from $8 \mathrm{GeV} / \mathrm{c}(\rho)$ to $15 \mathrm{GeV} / \mathrm{c}\left(K^{*}, \phi\right)$. An example of invariant mass spectrum for the $K^{* 0}(892)$ is shown in Fig. 目(right).

\section{Correlations}

Collective features of the Quark Gluon Plasma will be studied by observing various aspects of collective flow, including radial flow, directed flow and elliptic flow; also two-particle momentum correlations (HBT interferometry, also known as Femtoscopy) will provide crucial information about the size and shape of the fireball, while event-by-event fluctuations in several observables related to temperature, entropy, energy density, net charge, etc., will address the nature of the phase transition from Hadron Matter to the QGP.

Elliptic flow $\left(v_{2}\right)$ as a function of $p_{T}$ will be addressed by all three experiments. CMS will estimate the event plane using calorimeters and the inner tracker and will measure $v_{2}\left(p_{T}\right)$ using the event-plane, 2-particle correlations and cumulant methods [10]. ATLAS will use information from tracks, hits from inner tracker and energy in the first layer of calorimeters to measure $v_{2}\left(p_{T}\right)$ with three different methods [14], see Fig. B (left). ALICE will measure charged particle flow [6, 7] 

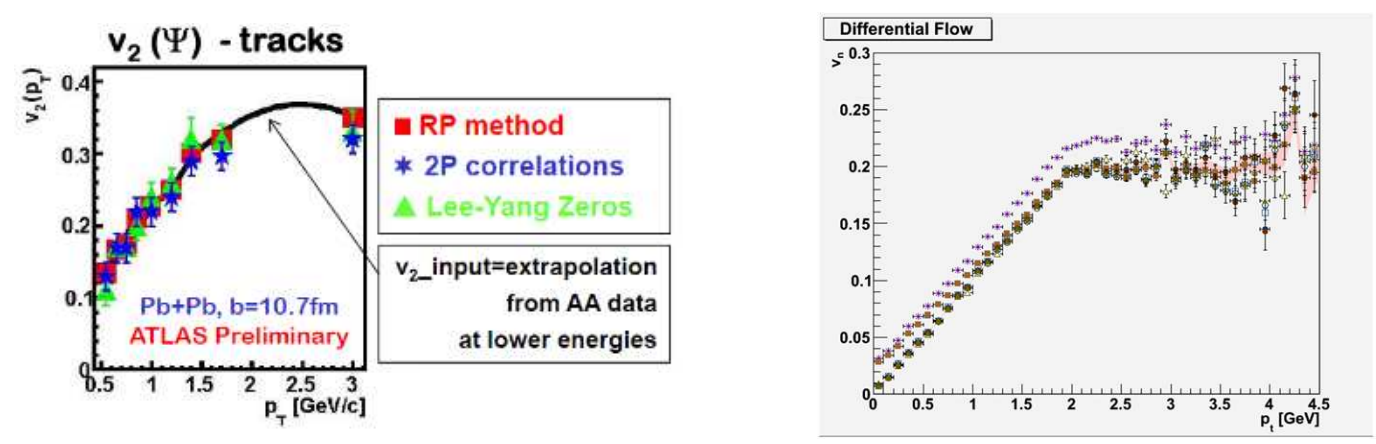

Figure 3: Left: Elliptic flow coefficient $v_{2}\left(p_{t}\right)$ as reconstructed from three different methods in ATLAS; right: $v_{2}\left(p_{T}\right)$ as reconstructed from several methods in ALICE: the scalar product and second order cumulants methods show deviations from MC input.

vs. $p_{T}$ with an even larger range of methods (see Fig. 3 right), which is crucial for the correct evaluation of non-flow effects; in addition, thanks to its excellent PID capabilities, ALICE will be able to measure flow separately for several particle species, which is a requirement to test among others the quark coalescence model.

ALICE will also address femtoscopy and fluctuations, for details see [6] and [7].

\section{Heavy flavours}

Heavy quarks (charm and beauty) are among the best "hard" probes for the medium produced in heavy ion collisions, for example the comparison of gluon, light quark and heavy quark energy loss as a function of $p_{T}$ will provide valuable information about the transport coefficients of the medium. In addition, due to their abundance, charm quarks may also display collective flow. The three experiments provide complementarity for the heavy flavour measurement because of their different acceptances in $\eta$ and $p_{T}$ and of different decay channels that can be studied.

The beauty decay channels studied so far include $B \rightarrow e+X, B \rightarrow \mu+X, B \rightarrow J / \psi+X \rightarrow$ $l^{+} l^{-}+X, B \rightarrow 5$ prongs, $(B) B \rightarrow \mu \mu+X$ as well as tagged b-jets. Charm is best studied via hadronic exclusive decay channels such as $D^{0} \rightarrow K^{-} \pi^{+}, D^{+} \rightarrow K^{-} \pi^{+} \pi^{+}, D_{s} \rightarrow K^{-} K^{+} \pi^{+}, D^{*} \rightarrow$ $D^{0} \pi, D^{0} \rightarrow K \pi \pi \pi$ and $\Lambda_{c} \rightarrow p K^{-} \pi^{+}$. Only a few channels will be mentioned in the following.

The $B \rightarrow e+X$ channel has been studied in ALICE [24] using electron identification in the TRD, which removes most hadrons, together with a cut on the impact parameter which removes electrons from charmed and strange particles as well as conversions and Dalitz decays: the $p_{T}$ spectrum of electrons from beauty can then be extracted up to $p_{T}$ of $20 \mathrm{GeV} / \mathrm{c}$. Beauty decay to $J / \psi$ has been studied both in ALICE and CMS. The distance from primary to secondary vertex is crucial to separate prompt from B-decay $J / \psi$ 's in both experiments; the minimum lepton $p_{T}$ will be $1 \mathrm{GeV} / \mathrm{c}$ in ALICE (electrons in the barrel) and $5 \mathrm{GeV} / \mathrm{c}$ in CMS (muons in the barrel).

Dimuons coming from semileptonic decays of at least one beauty hadron have been studied both in CMS (in the barrel) and in ALICE (in the forward muon spectrometer). CMS will use vertexing to separate dimuons coming from beauty from the Drell-Yan background, see Fig. Đ(left), while ALICE even without vertexing will be able to extract the beauty signal which is dominant in the single muon $p_{T}$ spectrum between 3 and $25 \mathrm{GeV} / \mathrm{c}$. 

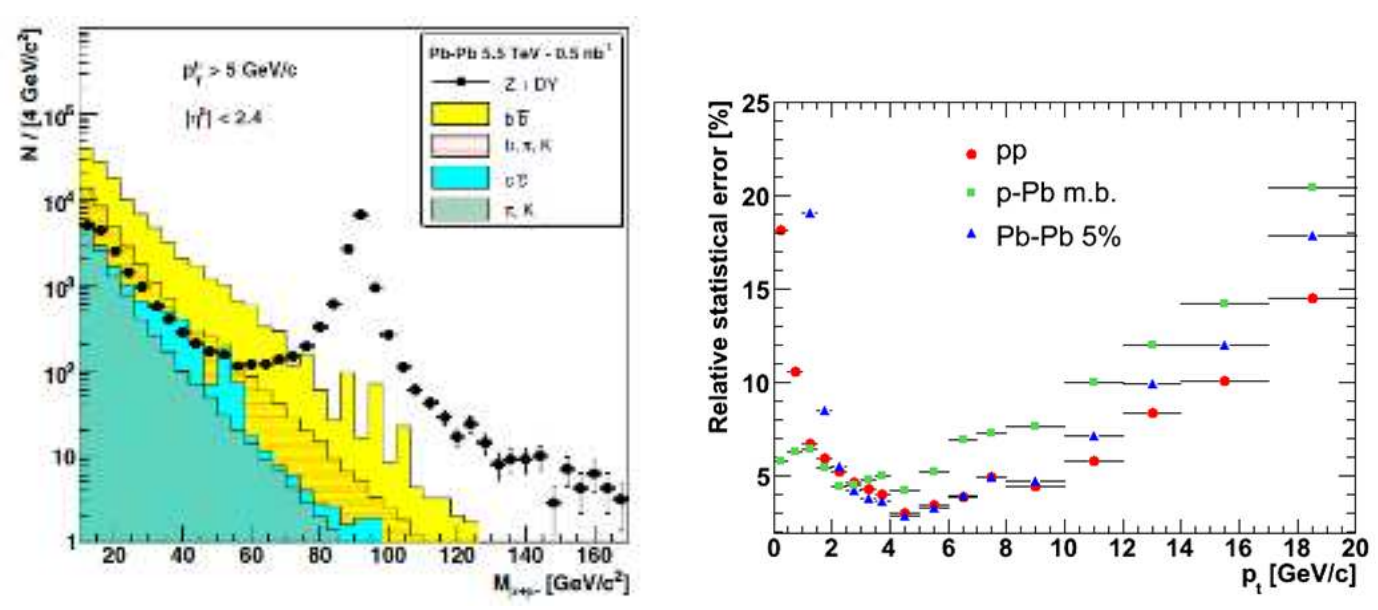

Figure 4: Left: different contributions to the dimuon spectrum, including the one from beauty decays, as simulated in CMS; right: relative statistical errors vs. $p_{T}$ for $D^{0}$ 's simulated in $\mathrm{pp}, \mathrm{p}-\mathrm{Pb}$ and $5 \%$ most central $\mathrm{Pb}-\mathrm{Pb}$ collisions, as reconstructed in ALICE.

Several hadronic charm decay channels have been studied extensively in ALICE [6] 7]. For example, the $D^{0} \rightarrow K^{-} \pi^{+}$signal is enhanced over the combinatorial background (which is reduced by a factor $\approx 1000$ ) by imposing cuts on the pointing of the $D^{0}$ reconstructed momentum to the primary vertex and on the $K$ and $\pi$ impact parameters: the statistical precision achievable in a standard year of $\mathrm{pp}, \mathrm{p}-\mathrm{Pb}$ and $\mathrm{Pb}-\mathrm{Pb}$ running for the $p_{T}$ spectrum of $D^{0}$ 's is shown in Fig. $\sharp$ (right). The measurement of charm quark flow is also feasible in ALICE.

\section{Conclusions}

A rich physics programme has been developed by the ALICE, ATLAS and CMS experiments for the first $\mathrm{LHC} \mathrm{Pb}-\mathrm{Pb}$ run, which is expected to take place at the end of 2010. Analysis procedures have been developed and tested on the GRID so that a smooth and efficient running of the analysis after first heavy ion data is anticipated. Detectors have been partially commissioned with cosmic muons and are expected to be fully commissioned and calibrated after the protonproton run. Concerning bulk properties and correlations, the current picture built on RHIC results (gluon saturation, statistical hadronization, quark coalescence) will be tested at LHC energy (initial center-of-mass energy is expected to be 20 times the maximum one at RHIC). Heavy flavours (charm and beauty) will be copiously produced at LHC: with the help of several heavy flavour tagging techniques which have been developed, the energy loss of heavy quarks will be studied in detail. Given the readiness of both detectors and analysis techniques, a wealth of new results is expected from the first heavy ion run at LHC.

\section{References}

[1] L. Evans and P. Bryant (eds.), LHC Machine, JINST 3 (2008) S08001.

[2] S. Myers, contribution to this Symposium.

[3] R. Bailey, contribution to this Symposium. 
[4] A. Drees, contribution to this Symposium.

[5] ALICE Collaboration, ALICE: Physics Performance Report, Volume I, J. Phys. G: Nucl. Part. Phys. 30 (2004) 1517-1763.

[6] ALICE Collaboration et al., ALICE: Physics Performance Report, Volume II, J. Phys. G: Nucl. Part. Phys. 32 (2006) 1295-2040.

[7] The ALICE Collaboration, K. Aamodt et al., The ALICE experiment at the CERN LHC, JINST 3 (2008) S08002.

[8] The ATLAS Collaboration, Heavy Ion Physics with the ATLAS Detector, LHCC 2004-009/1-013

[9] The ATLAS Collaboration, G. Aad et al., The ATLAS Experiment at the CERN LHC, JINST 3 (2008) S08003.

[10] The CMS Collaboration et al., CMS Physics Technical Design Report: Addendum on High Density QCD with Heavy Ions, J. Phys. G: Nucl. Part. Phys. 34 (2007) 2307-2455.

[11] The CMS Collaboration, S. Chatrchyan et al., The CMS experiment at the CERN LHC, JINST 3 (2008) S08004.

[12] P. Giubellino for the ALICE Collaboration, ALICE: Status and Perspectives, Proc. of HEP 2009, Krakow, Poland, PoS (EPS-HEP 2009) 027.

[13] D. Elia for the ALICE Collaboration, First measurements with the ALICE detector at LHC, Proc. of HEP 2009, Krakow, Poland, POS (EPS-HEP 2009) 042.

[14] The ATLAS Collaboration, A. Trzupek et al., Heavy Ion Physics with the ATLAS Detector at the LHC, Proc. of HEP 2009, Krakow, Poland, POS (EPS-HEP 2009) 043.

[15] I.A. Calì for the CMS Collaboration, CMS Experiment at LHC: Detector Status and Physics Capabilities in Heavy Ion Collisions, Proc. of HEP 2009, Krakow, Poland, PoS (EP S-HEP 2009) 044 .

[16] O. Kodolova, contribution to this Symposium.

[17] P. Giubellino, Heavy Ion Physics at the LHC, Proc. of HCP 2008, eConf C080527 (2008) [arXiv:0809.1062 (nucl-ex)]

[18] The ATLAS Collaboration, A. Trzupek et al., Global Observables for $\mathrm{Pb}+\mathrm{Pb}$ Collisions from the ATLAS experiment, Proc. of PANIC 2008, Eilat, Israel, ATL-PHYS-PROC-2009-021.

[19] N. Armesto et al., Relating High-Energy Lepton-Hadron, Proton-Nucleus, and Nucleus-Nucleus Collisions through Geometric Scaling, Phys. Rev. Lett. 94 (2005) 022002.

[20] The CMS Collaboration, CMS_PAS-QCD-07-001.

[21] The CMS Collaboration, CMS_PAS-QCD-09-002.

[22] J. Rafelski and B. Muller, Strangeness Production in the Quark-Gluon Plasma, Phys. Rev. Lett. 48 (1982) 1066 and Erratum, Phys. Rev. Lett. 56 (1986) 2334.

[23] I. Belikov for the ALICE Collaboration, Strangeness and heavy flavours within the ALICE experiment, Proc. of Strangeness in Quark Matter 2009, Sept. 27 - Oct. 2, 2009, Buzios, Brazil.

[24] A. Dainese for the ALICE Collaboration, ALICE Commissioning Status and Prospects to Measure Charmonium, Bottonium and bbar Production, Proc. of Beauty 2009, Sept. 7-11 2009, Heidelberg, Germany. 